\title{
EFFECT OF CURRENT DENSITY ON PROPERTIES OF NICKEL-SILICON CARBIDE COMPOSITES
}

\section{Kailash Hamal, Armila Rajbhandari (Nyachhyon), Gobinda Gyawali and Soo Wohn Lee}

Journal of Institute of Science and Technology

Volume 21, Issue 1, August 2016

ISSN: 2469-9062 (print), 2467-9240(e)

Editors:

Prof. Dr. Kumar Sapkota

Prof. Dr. Armila Rajbhandari

Assoc. Prof. Dr. Gopi Chandra Kaphle

JIST, 21 (1), 82-85 (2016)

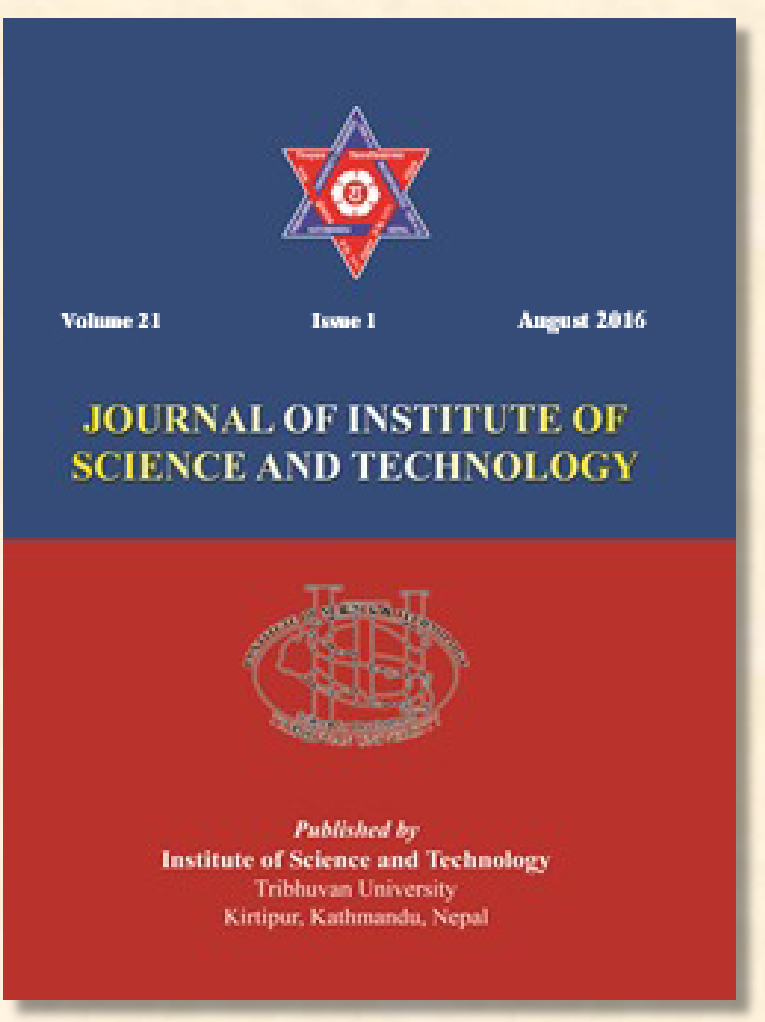

Published by:

Institute of Science and Technology

Tribhuvan University

Kirtipur, Kathmandu, Nepal 


\title{
EFFECT OF CURRENT DENSITY ON PROPERTIES OF NICKEL-SILICON CARBIDE COMPOSITES
}

\author{
Kailash Hamal $^{1}$, Armila Rajbhandari (Nyachhyon) $)^{1, *}$, Gobinda Gyawali ${ }^{2}$, and Soo Wohn Lee ${ }^{2}$ \\ ${ }^{1}$ Central Department of Chemistry, Tribhuvan University, Kirtipur, Nepal \\ ${ }^{2}$ Research Centre for Eco Multi-Functional Nano Materials, Global Research Laboratory, \\ Sun Moon University, Republic of Korea \\ *Corresponding email: armila3@yahoo.com
}

\begin{abstract}
Nickel-Silicon Carbide (Ni-SiC) composite coating has been prepared by two electrode electrochemical codeposition technique in nickel sulfamate bath. The Cetyltrimethylammonium bromide (CTAB) was added in bath as cationic surfactant whereas saccharine as a grain modifier. The effect of current density during deposition was systematically studied and optimized to get homogeneous surface texture with utmost microhardness and deprived coefficient of friction. The result revealed that $60 \mathrm{~mA} / \mathrm{cm}^{2}$ current density was found to be the optimum current density which showed the highest microhardness of $590 \pm 10 \mathrm{Hv}$ and lowest coefficient of friction with regular surface morphology.
\end{abstract}

Keywords: Ni-SiC; Morphology, Microhardness, Coefficient of friction

\section{INTRODUCTION}

Surface modification of metallic body by coating has become an essential process to improve the surface properties such as wear, corrosion and oxidation. Different techniques have been utilized for depositing organic as well as inorganic material onto the substrate to achieve desired properties. Composite electroplating is one of the methods that involve incorporation of metallic, non-metallic or polymeric micro or nano sized second phase particles along with the growing metal matrix. Such incorporated particles improve the overall properties of the coatings such as adhesion, anti-corrosion, anti-friction, microhardness and the mechanical strength. In recent years, the nickel based composite has been studied frequently to overcome and improve the properties of coating. A large number of ceramics particles incorporated with nickel matrix have also been reported in literatures (Shi et al., 2005, Gyawali et al., 2010, Garcia-Lecina et al., 2012). Besides this, $\mathrm{SiC}$ particles have also been extensively studied due to the cost effectiveness and wider applications (Shi et al., 2005, Gyawali et al., 2010, Garcia-Lecina et al., 2012).

Nickel sulfamate is a commonly used electrolyte during the electroplating of nickel due to its high deposition rate and superior throwing power. Addition of additives like Saccharine and CTAB along with sulfamate electrolyte helps to form smooth surface morphology by grain modification and increases co- deposition of particles, respectively (Shi 2005). In addition to this, during electroplating, operating conditions like $\mathrm{pH}$, temperature (Gyawali 2010, Hamal 2014), stirring rate (Hamal 2014, GarciaLecina 2012) and applied current has also played important role to obtain good quality deposit. In the previous study, we have presented the effects of additives on the $\mathrm{SiC}$ co-deposition into the nickel matrix. Different operating conditions like optimum temperature, stirring rate were also studied for the better surface texture, microhardness and tribological properties of the coatings (Gyawali et al., 2010, Hamal et al., 2014). In this study, Ni-SiC composite coatings have been prepared by using nickel sulfamate bath containing SiC nanoparticles, CTAB and saccharine as additives. The different current densities has been applied to find out the optimum current density, by keeping all other parameters such as pulse frequency and pulse duty cycle, constant. Thus prepared Ni-SiC coating samples were characterized and compared to get optimum current density.

\section{MATERIALS AND METHODS}

All chemical reagents were of analytical grade and obtained from Duksan Pure Chemicals Co. Ltd. Korea. These reagents were prepared in de-ionized water. Nickel sulfamate electroplating bath was formulated by taking $300 \mathrm{~g} / \mathrm{L}$ of $\mathrm{Ni}\left(\mathrm{NH}_{2} \mathrm{SO}_{3}\right)_{2}, \quad 10$ $\mathrm{g} / \mathrm{L}$ of $\mathrm{NiCl}_{2}$, and $40 \mathrm{~g} / \mathrm{L}$ of boric acid. In the bath, 0.2 
$\mathrm{g} / \mathrm{L}$ of CTAB was used as cationic surfactant while 2 $\mathrm{g} / \mathrm{L}$ saccharine has been used as a grain modifier. Similarly, $20 \mathrm{~g} / \mathrm{L}$ of $\mathrm{SiC}$ particle $(\sim 270 \mathrm{~nm})$ was also added into the bath. Then, nickel balls inside a titanium basket was taken as anode whereas SUS304 stainless steel of exposed area $6 \mathrm{~cm}^{2}$, was used as cathode. The co-deposition process was then carried out at $\mathrm{pH} 4.2$, temperature $50^{\circ} \mathrm{C}, 25 \%$ duty cycle, and $100 \mathrm{~Hz}$ of pulse frequency. In order to investigate the effect ofcurrent density during deposition, three different samples namely, S-20, S-60, S-100 were prepared at $20 \mathrm{~mA} / \mathrm{cm}^{2}, 60 \mathrm{~mA} / \mathrm{cm}^{2}$ and $100 \mathrm{~mA} / \mathrm{cm}^{2}$ current densities, respectively. The electrolytic bath was continuously stirred by using teflon coated magnetic stirrer at $250 \mathrm{rpm}$.

Scanning Electron Microscopy (Mini-SEM, Nanoeye) was used to examine the surface morphology and microstructure of the coatings, X-ray diffraction (XRD, Rigaku DMAX 2200, X-ray diffractometer
Japan) with $\mathrm{Cu}-\mathrm{K} \alpha$ wave length of $1.5 \AA$ was used to determine the crystallinity and phase present in the electrodeposited Ni-SiC coatings. XRD measurement was performed at the $2 \theta$ range of 10-100 degrees with the scan rate of $4 \% \mathrm{~min}$. The hardness of the composite coating was measured by Vickers microhardness tester (Buehler Ltd. USA) by applying 100g load for 10 seconds. Coefficient of friction of the prepared samples were recorded by ball on disc method using Tribometer (CSM Swiss) without using any lubricants in unidirectional sliding wear for $10 \mathrm{~min}$ under $2 \mathrm{~N}$ applied load.

\section{RESULTS}

\section{Surface Morphology}

Surface morphology of Ni-SiC composite coatings prepared at different current densities are shown in Fig.1.
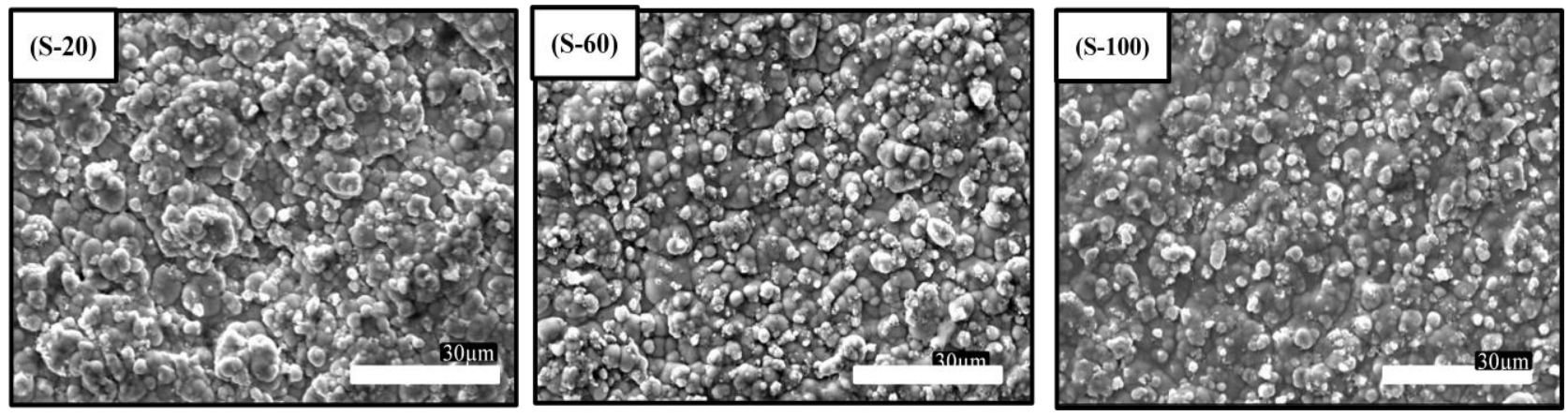

Fig.1. Surface morphology of Ni-SiC composite coatings prepared at different current density: sample (S-20) prepared at $20 \mathrm{~mA} / \mathrm{cm}^{2}$, sample $(S-60)$ prepared at $60 \mathrm{~mA} / \mathrm{cm}^{2}$ and sample $(S-100)$ prepared at $100 \mathrm{~mA} / \mathrm{cm}^{2}$.

\section{X-ray Diffraction (XRD) studies}

$\mathrm{X}$-ray diffraction patterns of the Ni-SiC composite coatings are shown in Fig. 2.

\section{Microhardness}

Hardness is one of the important properties of the coating that affects the application of the coatings in engineering purposes. Hence, the Vicker's microhardness of the Ni-SiC composite coatings were investigated for the samples prepared at different current densities. Fig. 3 shows the Vicker's microhardness plot of prepared samples.

\section{Coefficient of Friction}

Coefficient of friction was measured by unidirectional sliding wear in absence of lubricants for $10 \mathrm{~min}$ under the load of $2 \mathrm{~N}$. The variation of the coefficient of friction of $\mathrm{Ni}-\mathrm{SiC}$ coatings prepared at different current densities is presented in Fig. 4.

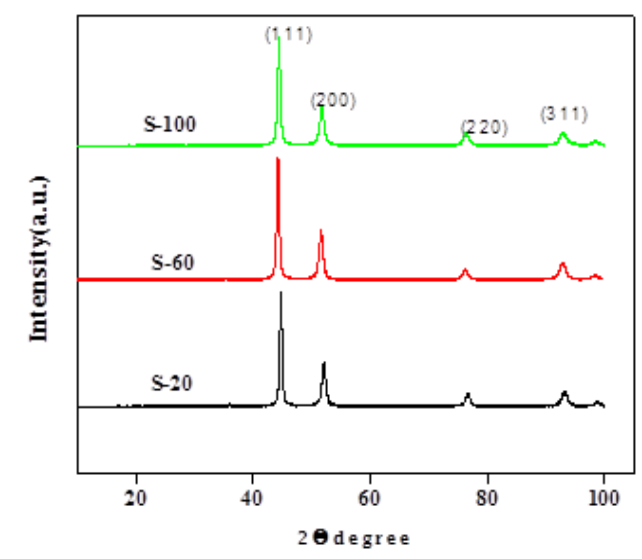

Fig.2: XRD patterns of $\mathrm{Ni}$-SiC composite coatings prepared at different current density: (S-20) prepared at $20 \mathrm{~mA} / \mathrm{cm}^{2},(S-40)$ prepared at 40 $\mathrm{mA} / \mathrm{cm}^{2}$ and $(S-100)$ prepared at $100 \mathrm{~mA} / \mathrm{cm}^{2}$. 


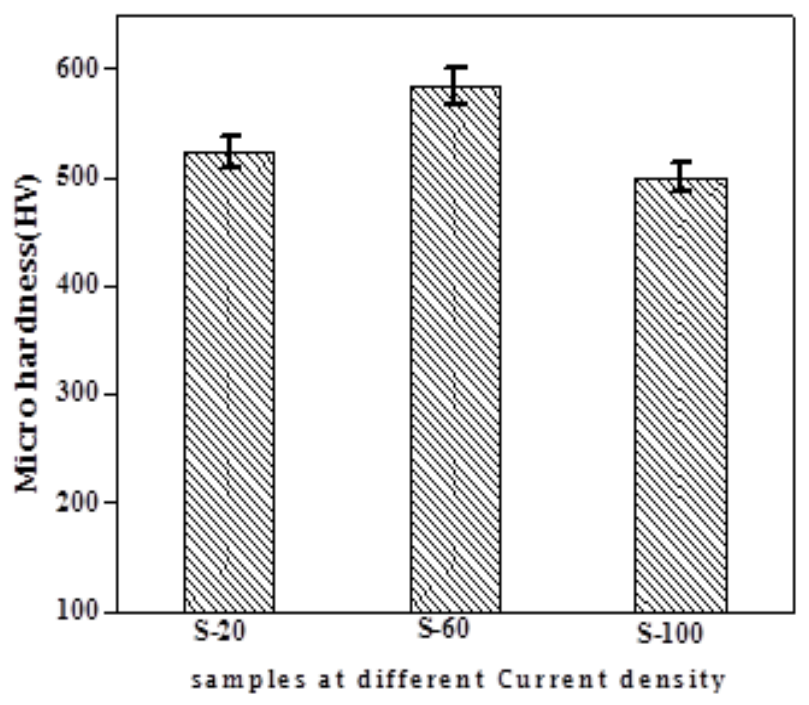

Fig.3: Vickers Microhardness of Ni-SiC composite coatings prepared at different current density: $(S-20)$ prepared at $20 \mathrm{~mA} / \mathrm{cm}^{2},(S-60)$ prepared at $60 \mathrm{~mA} / \mathrm{cm}^{2}$ and $(S-100)$ prepared at $100 \mathrm{~mA} / \mathrm{cm}^{2}$.

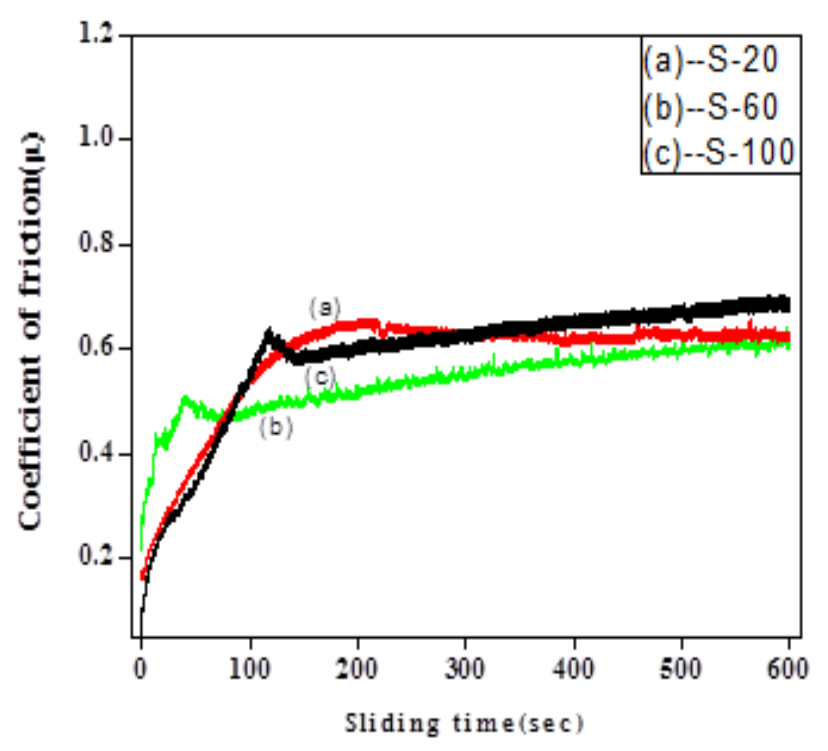

Fig.4: Coefficient of friction as function of time of $\mathrm{Ni}$-SiC composite coatings prepared at different current density: $(S-20)$ prepared at $20 \mathrm{~mA} / \mathrm{cm}^{2}$, $(S-$ 60) prepared at $60 \mathrm{~mA} / \mathrm{cm}^{2}$ and $(S-100)$ prepared at $100 \mathrm{~mA} / \mathrm{cm}^{2}$.

\section{DISCUSSION}

The study of morphology of the Ni-SiC coatings (Fig. 1) showed that at the lower current density, the Ni-SiC coatings possessed the larger grains at its surface and the grains were not uniformly distributed. On the other hand, the regular and homogeneous surface morphology was observed when the current density was set to higher. This result is attributed due to the faster deposition rate of $\mathrm{Ni}$ at the cathode and the increased co-deposition of second phase $\mathrm{SiC}$ particles onto the growing nickel matrix. The co-deposition of $\mathrm{SiC}$ particles lead to the breakage of the regular nickel grain and hence the alternative nucleation takes place. Thus, the process ultimately turned into the regular and homogeneous surface morphology at higher current densities.

On the basis of XRD pattern (Fig. 2), all the coatings exhibited a single phase on Ni matrix with FCC structure according to the JCPDS no. 04-0850. The relative peak intensity of (111), (220), and (311) peaks have been found to be increased by increasing the current densities up to $60 \mathrm{~mA} / \mathrm{cm} 2$. However, at $100 \mathrm{~mA} / \mathrm{cm}^{2}$, a slight decreased intensity of these peaks were observed. The elevation of these peaks correspond to the mixed [211] orientation of nickel crystallites favored in presence of incorporated $\mathrm{SiC}$ particles (Gyawali et al., 2010). Meanwhile, the decreased peak intensity of (200) reflection peak suggests the less preferred [100] texture orientation of Nickel. Hence, at higher current densities XRD analysis showed that, , there is mixed orientation of nickel deposits. It indicates the higher incorporation of second phase $\mathrm{SiC}$ nanoparticles.

Similarly, it was found that the sample S-60 prepared at $60 \mathrm{~mA} / \mathrm{cm}^{2}$ showed the highest value of Vickers microhardness $\sim 590 \mathrm{Hv}$ than the other two samples. Sample S-20 and sample S-100 showed relatively low value of Vicker's microhardness.

The outcome was explained on the basis of incorporation of $\mathrm{SiC}$ nanoparticles, grain modification and the textural orientation of nickel crystallites. The presence of well dispersed SiC nanoparticles in the nickel matrix enhances the load bearing capacity and restricted the propagation of slip planes. In addition, it is well known that the microhardness is also related to the grain size of the matrix. As the matrix grain size decreases, the microhardness increases. Similarly, [211] texture orientation was relatively harder in comparison to the preferred [100] orientation of the matrix. Therefore under the optimum condition, the electro-deposition of $\mathrm{Ni}$ $\mathrm{SiC}$ composite coating, favored by all the mentioned factors, collectively contributed to the enhancement of microhardness of the coatings.

The coefficient of friction shows the significant variations depending on both the sliding time and 
samples. Coefficient of friction of all the samples increased at the initial $2 \mathrm{~min}$ and reached to the steady state. It has been observed that the coefficient of friction of the samples S-20 and S100 were higher in comparison to the S-60 sample. The reduced coefficient of friction of S-60 sample is due to the well compact fine surface, higher microhardness value and the increased codeposition of $\mathrm{SiC}$ nanoparticles at this particular current density in comparison to the other samples. On the other hand, uniform dispersion of $\mathrm{SiC}$ nanoparticles in the nickel matrix may obstruct the ploughing out of larger flakes during the sliding wear. The formation of larger flakes due to the slippage of planes not only increases the frictional force but also cause to severe wear of the coating. Hence, the dispersed $\mathrm{SiC}$ nano particles might block the motion of dislocation and crack propagation in the soft matrix there by reduced the coefficient of friction of S-60 sample

\section{CONCLUSION}

In this investigation, the effect of variable current densities on the properties of $\mathrm{Ni}-\mathrm{SiC}$ composite coating was carried out. It has been found that the $\mathrm{Ni}-\mathrm{SiC}$ composite coating prepared at $60 \mathrm{~mA} / \mathrm{cm}^{2}$ exhibited the regular and smooth surface morphology. Similarly, the enhanced Vickers microhardness and the lower coefficient of friction were observed in the sample. Hence, from the morphological, microstructural, mechanical and tribological characterizations, the optimum current density was found at $60 \mathrm{~mA} / \mathrm{cm}^{2}$.

\section{ACKNOWLEDGEMENTS}

Kailash Hamal thanks the University Grants Commission (UGC) Nepal for partial financial support and Research Centre for Eco
Multifunctional Nano Materials, Global Research Laboratory, Sun Moon University, Korea for providing all laboratory facilities and financial support as well.

\section{REFERENCES}

Garcia-Lecina, E., Garcia-Urrutia, I., Diez, J. A., Morgiel, J., Indyka, P., 2012. A comparative study of the effect of mechanical and ultrasound agitation on the properties of electrodeposited $\mathrm{Ni} / \mathrm{Al}_{2} \mathrm{O}_{3}$ nanocomposite coatings. Surface and Coatings Technology, 206: 2998-3005.

Gyawali, G., Cho, S. H., Woo, D., Lee, S. W., 2010. Electrodeposition of Ni-SiC Nano Composite in Presence of Ultrasound. Materials Science Forum, 658: 424-427.

Gyawali, G., Hamal, K., Joshi, B., Rajbhandari, A., Lee S.W. 2014. Microstructural and electrochemical analysis of $\mathrm{Ni}-\mathrm{SiC}$ composite coatings prepared in presence of additives. Materials letters, 126: 228-231

Hamal, K., Gyawali, G., Rajbhandari, A., Lee S.W. 2014. Effect of Bath Temperature on Electrochemical Codeposition of Nickel Silicon Carbide composite. International Journal Chemistry and Pharmaceutical Sciences, 2(4): 658-663.

Hamal, K., Rajbhandari, A., Gyawali, G., Lee S.W. 2014. Effect of stirring rate on electrochemical codeposition of $\mathrm{Ni}-\mathrm{SiC}$ composite. Scientific World 12: 30-33.

Shi L., Sun C.F., Zhou F., Liu W.M., 2005. Electrodeposited nickel-cobalt composite coating containing nano-sized $\mathrm{Si}_{3} \mathrm{~N}_{4}$. Materials Science and Engineering A, 397: 190-194. 